\title{
Intelligent Decision Support for Business Workflow Adaptation due to Subjective Interruption
}

\section{Kohei Sugawara(1) ${ }^{1}$ and Hamido Fujita(2)}

(1) Sangikyo Corporation 4509, Ikebe-cho, Tsuzuki-ku, Yokohama, 224-0053, Japan, e-mail: sugawarak@ sangikyo.co.jp

(2) Iwate Prefectural University, Faculty of Software and Information Science, 152-52 Aza-Sugo, Takizawa, Iwate-gun, Iwate, 020-0193, Japan, e-mail: issam@iwate-pu.ac.jp

\begin{abstract}
Interruption is occurred by partial participation of workflows with adding new activity to a worker. A concerned worker to the interrupted workflow must restructure task assignment to improve consumed operating time by interruption. An opportunity of decision making is occurred to the workers to select appropriate tasks. However, preassignment of tasks to workers includes an expectation of superior decision maker to a training of an inexperienced worker. In order to avoid overmuch improvement to the interrupted workflow, the alternatives for shortening operating time of tasks is computed by TOPSIS method. Although positive ideal solution and negative ideal solution in conventional TOPSIS method are based on objective attributes, subjective completion time of pre-assigned tasks that are positive ideal solution in this model is depending on subjective attributes of the worker. Therefore, this research proposes a novel extension of positive and negative ideal solution of TOPSIS method by aggregating based on subjective fuzzy attributes of the worker and objective attributes of tasks. The approach is examined on simple case study showing that the work is promising on providing solution to handle interruption on carried work in runtime.
\end{abstract}

Keywords: Interruption; Workflow; Decision making; Subjective attributes; TOPSIS

1 This research is done through the $1^{\text {st }}$ author enrollment as Doctor student, the results achieved is part of the Doctor dissertation at Iwate Prefectural University, Japan supervised by Prof. Hamido Fujita. 


\section{Introduction}

In a working environment, opportunity of decision making by worker is occurred to generate higher profit or to eliminate severe loss. Herbert A. Simon defined three decision making processes that are Intelligence Activity, Design Activity and Choice Activity [1]. According to this definition, the worker who is a decision maker for operating assigned tasks must retrieve information to formulate alternatives by analyzing context of related tasks. The worker chooses appropriate decision after evaluating developed alternatives. Context of target tasks selected by the worker consists of objective attributes that are gettable several innate profits by task operation and costs to achieve pre-assigned objective. The worker selects appropriate task according to multi criteria of objective attributes that each task has. Tasks for getting profits are assigned to the workers depending on objective of organization as criteria for appropriate task selection of the worker by superior decision maker who could be manager, project leader or customer. Assigned tasks to the workers are structured to get profits for belonging organization of the workers by steps as workflow through consideration of experts. The worker has subjective attributes for task operation based on worker's profile such as experience or operational skill. Degree of expected profits by operating task is changed by applying subjective attributes of the worker to objective attributes of task through task assignment. Expected profits for the organization by assigning tasks are increased by improving work efficiency of current work or forthcoming work. Work efficiency of current work is improved by reducing labor cost that is engagement time of the worker to the assigned task while keeping achievement of the task. Completion time of task as engagement time of the worker is shortened by assigning experienced worker. Although the completion time of task by inexperienced worker is longer than experienced worker, applicable experience for forthcoming similar task is accumulated with time by engaging to the task. Therefore, shortening time for improvement of work efficiency in current task and experience to forthcoming work are trade-off. Weights of each criterion for the appropriate task selection are affected dynamically by an external interference to the worker. The external interference to the worker generates new activity to current workflow of the worker. Corragio (1990) defines an interruption as an "externally-generated, randomly occurring, discrete event that breaks continuity of cognitive focus on a primary task" [2]. Interruption of operation continuity by the worker on the workflow is occurred by invoking new or updates another task of the worker. In other words, interruption is occurred by partial participation of multiple workflows. Interruption would produce positive or negative effect to the worker and the current workflow. Interruption is formalized through two parametric views that are time and context. The time parametric view of interruption is related to operating time of the generated task and completion time of interrupted workflow. The degree of positive or negative effect to forward the current workflow can be represented by normalizing time of the interruption. The context parametric view of interruption 
is related to operation and process to the current workflow. The context of the generated task by interruption is analyzed to formulate alternatives based on influenced elements of interruption. Although interruption by non-task such as taking rest or enjoying hobby has positive effect to efficiency of the current workflow by improving mental or physical tiredness, any interruption generates negative effect to the current workflow in time parametric view point. Starting time of subsequent tasks on the interrupted workflow is delayed by consuming time for the interruption. Each weight of criteria for selecting appropriate task is changed to improve the delay of the current workflow by re-assigning tasks of inexperienced worker to experienced worker. There are many approaches for improving work efficiency by handling resources of workers such as job scheduling problem or flow shop scheduling [3], [4]. Although conventional scheduling problem approaches can optimizes work efficiency by scheduling, reduction of experience for inexperienced workers due to assigning tasks to experienced worker are not discussed. Task assignment for improvement of too higher efficiency decreases work opportunity of inexperienced worker. The expected profits of pre-assignment for forthcoming work are reduced by the negative effect of interruption and overmuch improvement by re-assigning tasks. In order to minimize the payoff of expected profit for forthcoming work, the appropriate task selection of the worker in interruption must approximate to the expected profit by pre task assignment as an ideal task selection through the superior decision maker. Leaving time (disengagement) of the current workflow due to interruption generates negative effects to short term memory of the interrupted worker because of forgetting the task of workflow. Memory of the interrupted worker for the interrupted work is reduced with time. Relation of both time and degree of decreasing memory is represented by Ebbinghaus's Forgetting Curve [5]. Negative effect of the lacked working memory is appeared when past task is resumed from added tasks. By leaving from the interrupted work, usable time for the interrupted workflow is consumed and accuracy of decision making for the interrupted work is decreased [6]. Experienced worker can decide appropriate task according to own judgment heuristics [7]. The accuracy of decision making by experienced worker also is reduced after long time interruption. Therefore, the authenticity of experienced worker's decision making for selecting appropriate tasks of the current workflow is decreased by interruption. Previously, authors discussed about decision support system algorism for generated task by estimating interruption context according to working history and by using knowledge mining [8]. The previously proposed system assists operation of new task to reduce time consuming for interruption by recommending appropriate alternatives. In addition to the above issue, concerned workers of the interrupted workflow must restructure operation for the workflow according to the effected situation by interruption. The section 2 is shown the outline of the proposed approach for supporting decision making of appropriate task selection of worker. 


\section{Proposed Approach}

Decision making, for the appropriate task selection in interruption, is improvement of affected work efficiency to the current workflow caused by interruption. Effective alternatives for concerned workers of the interrupted workflow can be ranked according to multi criteria for related tasks, for example through AHP (Analytical Hierarchical Process) [9] or other techniques [10] [11]. The attributes for weighting criteria in the interruption model are classified into two aspects; objective attributes and subjective attributes that are based on nature of the attributes. The objective attributes for weighting the criteria means static objective of organizations. A worker who is employed by organization is oriented to objective of the organization by company strategy. The objective of organization is aggregation of multi criteria attributes for stakeholders in a company. The criteria of task assignment by superior decision maker include work efficiency of workers for benefit of the organization and training for workers that leads to organization benefits in its progress. Meanwhile, the subjective attributes means dynamic changing work environment that consists of fuzzy elements of workers and tasks. Since completion time of tasks are depending on assigned worker's operational skill to task, the situation of interruption for operating workflow is consisted by using the subjective attributes of workers for related tasks. Therefore, the proposed weights of multi criteria are computed by aggregating the objective attributes for tasks aligned on the subjective attributes of workers. The interrupted workers must select appropriate task in dynamic work changing environment within the objective of organization. In addition, the concerned workers to the interrupted workflow must avoid the overmuch improvement to keep the expected profit for organization. There are following multi criteria decision making techniques for approaching alternatives to positive ideal solution. TOPSIS method (Technique for Order of Preference by Similarity to Ideal Solution) [12] can compute best solution to the worker in this situation by setting an ideal objective of an organization as positive ideal solution. Although VIKOR method (Vlse Kriterijumska Optimizacija Kompromisno Resenje) [13] also can rank by using positive ideal solution and negative ideal solution, appropriate alternatives in interruption must distance the negative ideal solution to keep the expected profit of inexperienced worker. Fuzzy TOPSIS [14] can extend crisp values of positive and negative ideal solution in traditional TOPSIS by weighting criteria as objective attributes. The objective attributes of the organization is aggregation of subjective attributes that is dependable on worker's experience (extracted from works preferences). Therefore, this research proposes a novel extension of positive and negative ideal solutions of TOPSIS method by aggregation based on subjective fuzzy attributes of the worker. There are approaches for setting ideal solution by aggregating individual decision making as group decision [15], [16]. Although consensus for appropriate decision making can be improved by including individual decisions as group decision, the proposed approach does not require multiple decisions because the ideal solution in 
interruption is computed based on the subjective attributes of workers. Alternatives for apropriate task selection in interruption must improve delay of workflow to achieve by deadline of the workflow. There is an approach to reduce the number of AGVs (automated guided vehicles) in container terminals without effect on completion time of workflow [17]. Cost of AGVs in this approach is reduced by computing appropriate order of the container quay crane and AGVs' operation on the basis of modeling by using the pseudo-analysis. Concerned workers in interruption already have tasks for achieving workflow by deadline. Therefore, appropriate order of tasks for improving delay as alternatives must includes individual difference of concerned workers' skills as subjective attributes. The consumed time for the current workflow due to interruption can be improved by supporting tasks of inexperienced worker by the experienced worker as formulated alternatives. The improvable subjective completion time of inexperienced worker's task is changed according to degree of supporting worker's experience. Therefore, the proposed alternatives for appropriate task selection in interruption are formulated by computing subjective completion time of task based on experience of workers. The scores of proposed alternatives for positive or negative ideal solution are computed by estimating expected experience of workers in task operation. Each expected experience of alternatives is computed based on proportion of engagement of experienced worker for improving the consumed time by interruption. Operating time of the inexperienced worker is shortened by asking related information for operating task to the experienced worker without gathering information by oneself. Although the subjective completion time of inexperienced worker is shortened by increasing engaging time of the experienced worker for the task of the inexperienced worker, expected experience of workers by pre-assignment as positive ideal solution is decreased by reducing working opportunity of the inexperienced worker. Therefore, appropriate alternatives for the expected experiences by pre-assignment are ranked according to reduced expected experiences to achieve required engaging time of supporting worker for tasks of the interrupted worker. The distance of proposed alternatives to the positive ideal solution is approached by reducing the engaging time of experienced worker for the task of inexperienced worker. The engaging time for supporting each task is changed based on subjective completion time of the experienced workers for each task. The subjective completion time of the supporting worker for improving negative effect by interruption is shortened by giving related information to the task from documentation of the experienced worker to the supporting worker. Document for knowhow is reflected by experience of document creator. Expertise of the document to the task is extracted according to similarity of the context of the task and the document. The expected experience of alternatives is reduced by shortening the subjective completion time of each task of the interrupted worker with the similar document. Since the distance of the positive ideal solution's score and the score of alternatives is shortened by reducing the required engaging time of supporting worker for the tasks of the interrupted worker, the ranking of 
appropriate task selection for shortening negative effect of interruption is changed depending on degree of improvement for the tasks by the similar document. In order to recommend appropriate task selection within the negative effect to the workflow caused by interruption, proposed approach utilizes TOPSIS method to rank appropriate alternatives for the interrupted worker by aggregating objective attributes of task and subjective attributes of worker with objective attributes of related documentations. In our approach the workflow is given. Our purpose is to have the execution of the work be kept or optimized based on the interruptions context project by attributes consider as either objective or subjective ones.

\subsection{Definition of Target Tasks}

Workflow is an optimized representation of steps to achieve work objective by expert worker and specified in optimal manner based on business policy and etc. These steps are ordered as task by eliminating negative cause such as loss of time, duplicative process and so on. The divided task by steps is an action for achieving simple objective task as input of the other task. Task that needs results of the other task as input is ordered after completing the related task. Tasks that have no input or output relations can be reallocated in parallel. The parallel tasks on workflow can be assigned to multiple workers or multiple project members for operating tasks simultaneously. The task on the workflow is activity that needs to be accomplished within defined period of time or by deadline. Objective of each task is achieved by completing operation of worker before the deadline of each task. The length of usable time for the task operation of worker is up to the deadline of the task from the completion time of prior task. The task that has deadline of short period is ordered earlier than task of long period deadline. Entire deadline of the workflow is defined by aggregating estimated completion time of each task. Even if there is similar task in the workflow, order of these tasks is sequential based on different operating period. The order of tasks in the workflow can be changed by keeping the completion time of each task related deadlines. Therefore, selectable tasks for improving work efficiency by the concerned workers to the interrupted workflow are tasks that have finished the related prior tasks as inputs without including already finished tasks. Figure 1 shows matrix for pre-assignment (PreA) by the superior decision maker. Number of rows in the matrix of pre-assignment means assigned workers to same workflow. Each operating time $(O t)$ of task according to deadline of the task and operable time period of the assigned worker is substituted to each columns of assigned worker. The coordinates of nonassigned tasks has value of zero. 


$$
\operatorname{PreA}=\left(\begin{array}{ccc}
O t_{11} & \cdots & O t_{1 h} \\
\vdots & \vdots \\
O t_{g 1} & \cdots & O t_{g h}
\end{array}\right) \quad \begin{aligned}
& g=\text { number of workers to the workflow } \\
& h=\text { number of tasks to the workflow }
\end{aligned}
$$

Figure 1

Matrix for pre-assignment by superior decision maker

\subsection{Relation of Time and Experience}

Subjective completion time of task is changed depending on tasks' context and experience of workers. The context of task can be classified as physical work and mental work. The physical work is an activity based on physical condition of worker such body related problems, or muscular tension or physical change in office or working space. The physical part of the work is related to the objective attributes of task based an organization's objectives represented as criteria. Meanwhile, the mental work is an activity of information gathering for the steps of the task operation that lead to efficient physical work. Operating time for the mental work is related to searching and analyzing time based on individual experience of workers as subjective attributes. Experienced worker can shorten the time for the mental work by utilizing information for the steps of the task operation based on accumulated experience through a past task operation as knowledge. Workers acquire multiple types of experiences for the mental work depending on context of task through the task operation. The context of task is represented as a set of objective attributes by weighting of operating time of task to each type of experience. Impact for each type of experience on operating time of task can be weighted as fuzzy membership by experienced worker. The membership function of task for each type of experience is shown in following.

$$
\begin{array}{ll}
\sum_{i=1}^{l} \mu w(i)=1.0 & \mu w(i)=\text { weight of each type of experience } \\
l=\text { number of related experiences for task }
\end{array}
$$

Each value of accumulating experience with a time is evaluated as objective attributes of task by linguistic value of experienced worker. Amount of individual knowledge for workers as the subjective attributes is accumulated by multiplying defined weights of experience $(\mu w(i))$ and operating time of task. Accumulation of experience is higher for inexperienced worker according to learning curve [18]. In other words, expected experience for experienced worker from task operation is smaller than inexperienced worker. Subjective expected profit by task for each experience $(S P f(i))$ is computed as in the following expression. 


$$
\begin{array}{ll}
\operatorname{SPf}(i)=\frac{\left(O t_{g h} * \mu w(i)\right)}{\left(O t_{g h} * \mu w(i)\right)+W e(i)} & i=\text { type of experience } \\
W e(i)=W e(i)+\operatorname{SPf}(i) & W e(i)=\text { accumulated experience of worker }
\end{array}
$$

Operating time $(O t)$ for each task is defined by task assignment based on deadline of each task and operable period of workers. The subjective expected profit $(S P f(i))$ for experiences of the task assigned worker is decreased by increasing the past accumulated experiences $(W e(i))$ of the worker. Therefore, enhancement of operating task is maximized by assigning tasks of workflow to the inexperienced worker. The time for operating task has essential core time $(c t)$ and improvable time by the experienced worker. The core time $(c t)$ means essential engagement of the worker to the task that includes physical execution or waiting such as running time of machine. Experienced worker can shorten the time for engagement of thinking that includes information gathering for task achievement by utilizing their expertise. Therefore, the core time $(c t)$ that is impossible to shorten by experience is decided by the experienced worker. The subjective completion time $(S C t)$ of task that is changed by amount of experiences of the task assigned worker is computed by crossing the above defined objective attributes of the task to experience. Small expected experience from the task operation means the task assigned worker has related large amount of experience to the task. Impact (iSPf) of the time for the engagement of thinking is computed by averaging each subjective expected profit $(S P f(i))$ for the task assigned worker. Following expression represents the subjective completion time $(S C t)$ of the task with the worker.

$$
\begin{array}{ll}
i \operatorname{SPf}=\left(\sum_{i=1}^{l} \operatorname{SPf}(i)\right) / l & l=\text { number of related experiences for task } \\
S C t=c t+\left(i \operatorname{SPf} *\left(O t_{g h}-c t\right)\right) & c t=\text { core time for task operation }
\end{array}
$$

The subjective completion time other than the core time is computed by multiplying the impact ( $i S P f$ ) of subjective profits for the task assigned worker to the shortened operating time by experienced worker. The shortened operating time is computed by subtracting the core time from pre-assigned operating time $(\mathrm{Ot})$ based on deadline of the task. Therefore, the subjective completion time (SCt) is maximized by assigning the task to inexperienced worker for the task.

\subsection{Setting Positive Ideal Solution and Negative Ideal Solution}

Positive ideal solution for decision making of improving work efficiency by TOPSIS method is extracted from expected profits of workers by pre-assignment in the proposed approach. Decision of superior worker who assigned tasks is reflected to avoid the task selection of overmuch improvement for the current 
workflow by extracting from pre-assignment as positive ideal solution. Consensus of alternatives for improving effect of interruption is defined by aggregating the weighting of experienced worker and pre task assignment by superior decision maker of the current workflow. Target tasks for improving negative effect of interruption as alternatives on decision making are the confined tasks that have same deadline. The score of positive ideal solution $(P S)$ is computed as objective attributes by aggregating each subjective profit for experience of each worker from the target tasks by pre-assignment. Figure 2 shows matrix of the positive ideal solution $(P S)$ and negative ideal solution $(N S)$ in interruption.

$$
\begin{aligned}
& P S=\left(\begin{array}{ccc}
E_{11} & \cdots & E_{1 l} \\
\vdots & & \vdots \\
E_{k 1} & \cdots & E_{k l}
\end{array}\right) \\
& N S=\left(\begin{array}{ccc}
E_{11}^{\prime} & \cdots & E^{\prime}{ }_{1 l} \\
\vdots & & \vdots \\
E^{\prime}{ }_{k 1} & \cdots & E^{\prime}{ }_{k l}
\end{array}\right) \\
& E_{k l}=\sum_{i=1}^{n} \operatorname{SPf}_{1}(i) \\
& k=\text { number of concerned workers for interruption } \\
& l=\text { number of related experiences for task } \\
& n=\text { number of target tasks assigned to worker } \\
& E^{\prime}{ }_{k l}=\sum_{i=1}^{n} \operatorname{SPf}_{2}(i) \\
& S P f 1(i) \text { is based on pre-assignment } \\
& S P f 2(i) \text { is based on shortest total completion time }
\end{aligned}
$$

Figure 2

Matrix of positive ideal solution and negative ideal solution

The expected profits $\left(E_{k l}\right)$ of each experience to the task assigned workers are computed by aggregating same experience type of pre-assigned tasks that have same deadline. The number of concerned workers for interruption is selected according to the deadline of assigned tasks on the same workflow. In order to avoid overmuch improvement for the current workflow by restructuring of task assignment, the expected profits $\left(E^{\prime}{ }_{k l}\right)$ of task assignment that have shortest total subjective completion time of related workers within possible combination of task assignment in the interruption is computed as negative ideal solution (NS). Number of combinations for the possible task assignment is computed according to the number of workers and deadline of selectable tasks. Therefore, the alternatives that have total expected profits for forthcoming task than preassignment are included as improvement for the interruption.

\subsection{Context of Task and Documentation}

The context of task in proposed approach consists of the terms for classifying each type of experiences and the weighted values of profit to each experience as scalar value. Context of document that is created by experienced worker can be represented based on the used keywords for linguistic classification of the type of experience $(\mu w)$. Experts conclude the main criteria for workflow, and evaluate 
attributes in pair wise fashion based on their strategy etc. The context of document in knowledge base can be represented as vector by counting term frequency of each keyword to compute the relative importance weight of criteria. Therefore relevancy of the target tasks and document of experienced workers are computed with using terms as common features by comparing correlation of two vectors that are the context of the task and the context of the document. The correlation of the two vectors are compared to compute multiple terms of the two vectors on monotonic property by using cosine distance [19]. Figure 3 shows calculation of similarity by cosine distance.

$$
\begin{aligned}
& \vec{p}=\{\mu w(1), \mu w(2), \cdots, \mu w(j)\} \\
& \vec{q}=\{\operatorname{Tm}(1), \operatorname{Tm}(2), \cdots, \operatorname{Tm}(j)\} \\
& \operatorname{Cos}(\vec{p}, \vec{q})=\frac{\sum_{i=1}^{j}(\mu w(i) * \operatorname{Tm}(i))}{\sqrt{\sum_{i=1}^{j}(\mu w(i))^{2}} * \sqrt{\sum_{i=1}^{j}(\operatorname{Tm}(i))^{2}}}
\end{aligned}
$$

Figure 3

Computing similarity by cosine distance

The vector $p$ consists of proportion of weighted profit for the task $(\mu w(j))$ by classifying named experiences. $\operatorname{Tm}(j)$ of the vector $q$ for the documents are normalized to create the vector for analyzing by using term frequency meaning according to terms of the context of task. The similarity of two vectors is higher by approaching score of cosine distance to 1.0 .

\subsection{Formulating Alternatives with TOPSIS}

Appropriate alternatives for ideal expected profits by pre-assignment are formulated based on the subjective completion time of selectable tasks and generated task by interruption. Formulated alternatives must be able to negate to the expected consumed time $(E C t)$ by interruption. The expected consumed time $(E C t)$ is computed by computing the subjective completion time of the generated task by interrupted worker.

$$
E C t=c t+\left(i S P f *\left(O t^{\prime}-c t\right)\right)
$$

Operating time $\left(O t^{\prime}\right)$ for the generated task by interruption is an operable period of the interrupted worker by deadline of the task. Each worker who is concerned to the target tasks in interruption has subjective flexible time $(\operatorname{SFt}(i))$ for usable another tasks based on deadline of pre-assigned tasks and each subjective completion time. Total flexible time $(\operatorname{TSFt}(k))$ of the interrupted worker who has multiple tasks can be used for the expected consumed time $(E C t)$ by interruption.

$\operatorname{SFt}(i)=O t_{g h}-S C t(n)$ 
$\operatorname{TSFt}(k)=\sum_{j=1}^{n} \operatorname{SFt}(i) \quad \begin{aligned} & k=\text { number of concerned workers for interruption } \\ & n=\text { number of target tasks assigned to worker }\end{aligned}$

Achievement of tasks on the current workflow is failed by exceeding the expected consumed time $(E C t)$ from total flexible time $(\operatorname{TSFt}(k))$ of the interrupted worker. The subjective flexible time $(\operatorname{SFt}(n))$ of each worker also can be utilized to support interrupted worker's tasks. The exceeding time $(E E C t)$ of the interrupted worker is improved by consuming operating time of the other task within the subjective flexible time of the other worker. Therefore, alternatives $(\operatorname{Alt}(x))$ for improving the expected consumed time $(E C t)$ by interruption are formulated based on the supporting time of another worker for improving the exceeding time of the interrupted worker. The required time $(R t)$ for supporting the interrupted worker is computed based on proportion of exceeding time $(E E C t)$ for interrupted worker and the impact of subjective profits $(i S P f)$ for the experience of supported task by the workers.

$$
\begin{array}{ll}
E E C t=E C t-T S F t(k) & (E C t>\operatorname{TSF} t(k)) \\
E E C t: \operatorname{iSPf}(s)=R t: i \operatorname{SPf}^{\prime}(s) & \\
R t=E E C t * \frac{i \operatorname{SPf} f^{\prime}(s)}{i \operatorname{SPf}(s)} & i S P f(s) \text { by interrupted worker }
\end{array}
$$

The subjective expected profits $(\operatorname{SPf}(s))$ of supported task for interrupted worker by other worker is computed by subtracting the exceeding time (EECt) from the subjective completion time $(S C t(s))$ of the interrupted worker for the supported task as operating time. The subjective expected profits $(\operatorname{SPf}(i))$ of the other preassigned tasks for the interrupted worker is computed by using the subjective completion time $(S C t(i))$ of the interrupted worker. Total expected profits $\left(E_{a l}\right)$ for the interrupted worker who is supported by the other worker in alternatives is computed by aggregating expected profits of the pre-assigned tasks within total flexible time $(\operatorname{TSFt}(k))$ of the interrupted worker.

$$
\begin{aligned}
& \operatorname{SPf}(s)=\frac{((S C t(s)-E E C t) * \mu w(s))}{(\operatorname{SCt}(s) * \mu w(s))+W e(s)} \quad \begin{array}{c}
(W e(i)=W e(s)+S P f(s)) \\
s=\text { type of experience for the supported task }
\end{array} \\
& \operatorname{SPf}(i)=\frac{(\operatorname{SCt}(i) * \mu w(i))}{\left(\operatorname{SCt}(s)^{*} \mu w(s)\right)+W e(s)} \quad(W e(i)=W e(i)+\operatorname{SPf}(i)) \\
& E_{a l}=\operatorname{SPf}(s)+\sum_{i=1}^{n-1} \operatorname{SPf}(i) \quad \begin{array}{l}
l=\text { number of related experiences for task } \\
n=\text { number of target tasks assigned to interrupted worker }
\end{array}
\end{aligned}
$$

Meanwhile, the total subjective expected profits $\left(E_{b l}\right)$ for the supporting worker as the score of alternatives are computed by aggregating the subjective expected profits $(\operatorname{SPf}(s))$ of the supporting task and the subjective expected profits $(\operatorname{SPf}(i))$ of pre-assigned tasks to the supporting worker. The negative effect of interruption 
is improved by utilizing the subjective flexible time $(S F t)$ of the supporting worker as alternatives. Therefore, the total subjective flexible time (TSFt) of the supporting worker must be longer than the required time $(R t)$ for improving the exceeding time $(E E C t)$ of the interrupted worker. The subjective expected profit $(S P f(s))$ of the supporting worker for supporting task is acquired by computing the required time $(R t)$ and impact of subjective profits ( $i S P f)$ for the supported task.

$$
\begin{aligned}
& \operatorname{SPf}(s)=\frac{\left(R t^{*} \mu w(s)\right)}{\left(R t^{*} \mu w(s)\right)+W e(s)} \\
& \operatorname{SPf}(i)=\frac{\left(\left(O t_{g h}-S F t\right) * \mu w(i)\right)}{\left(\left(O t_{g h}-S F t\right) * \mu w(i)\right)+W e(i)} \quad \begin{array}{l}
(S F t<R t) \\
(W e(i)=W e(i)+S P f(i))
\end{array} \\
& \operatorname{SPf}(i)=\frac{\left(\left(O t_{g h}-R t\right) * \mu w(i)\right)}{\left(\left(O t_{g h}-R t\right) * \mu w(i)\right)+W e(i)} \quad \begin{array}{l}
(S F t>R t) \\
(W e(i)=W e(i)+S P f(i))
\end{array} \\
& E_{b l}=\operatorname{SPf}(s)+\sum_{i=1}^{n} \operatorname{SPf}(i) \begin{array}{l}
l=\text { number of related experiences for task } \\
n=\text { number of target tasks assigned to interrupted worker }
\end{array}
\end{aligned}
$$

The subjective expected profit $(S P f(i))$ of the supporting worker by preassignment is decreased by subtracting applied flexible time $(S F t)$ for supporting from operating time $\left(\mathrm{Otgh}_{g}\right)$ of the pre-assigned task. When the required time $(R t)$ for supporting the interrupted worker is longer than the subjective flexible time $(S F t)$ of the task, the required time $(R t)$ is consumed by utilizing the flexible time of the other tasks of the supporting worker. Distance of alternatives and positive ideal solution $(P S)$ on the expected profits $\left(E_{k l}\right)$ of each experience to the workers is shortened by shortening the required time $(R t)$ for the interrupted worker. The required time $(R t)$ for the interrupted worker is shorted by increasing an impact of subjective profits (iSPf') of the supporting worker for the supported task. The proposed approach shortens the required time $(R t)$ of the supporting worker for improving the exceeding time $(E E C t)$ of the interrupted worker by applying similar document to the supporting worker as experiences for task operation according to experiences $(D e(n))$ of document creator. Degree of applied experiences of document creator is computed based on a similarity $(\operatorname{Sm}(n))$ of tasks and documents. The impact of subjective profits (iSPf') for shortening the required time $\left(R t^{\prime}\right)$ with the similar document as alternatives are computed as follows.

$$
\begin{aligned}
& \operatorname{SPf}(s)=\frac{\left(O t_{g h} * \mu w(s)\right)}{\left(O t_{g h} * \mu w(s)\right)+(W e(s)+(\operatorname{De}(s) * \operatorname{Sm}(s)))} \\
& \operatorname{SSPf}^{\prime \prime}(s)=\left(\sum_{i=1}^{l} \operatorname{SPf}(s)\right) / l \quad l=\text { number of related experiences for task }
\end{aligned}
$$




$$
R t^{\prime}=E E C t * \frac{i \operatorname{SPf} f^{\prime \prime}(s)}{i \operatorname{SPf}(s)} \quad \begin{aligned}
& i \operatorname{SPf} f^{\prime \prime}(s) \text { by supporting worker with similar document } \\
& i \operatorname{SPf}(s) \text { by interrupted worker }
\end{aligned}
$$

The alternatives with the similar document shorten only engaged time of thinking such as information gathering. Therefore, the experiences of the document creator are not added to the expected profits of alternatives. Figure 4 shows matrix of alternatives for improving the exceeding time $(E E C t)$ of the interrupted worker. The scores for concerned workers who do not include the interrupted worker and the supporting worker are substituted same value of the positive ideal solution $(P S)$ to the matrix of alternatives $(\operatorname{Alt}(x))$.

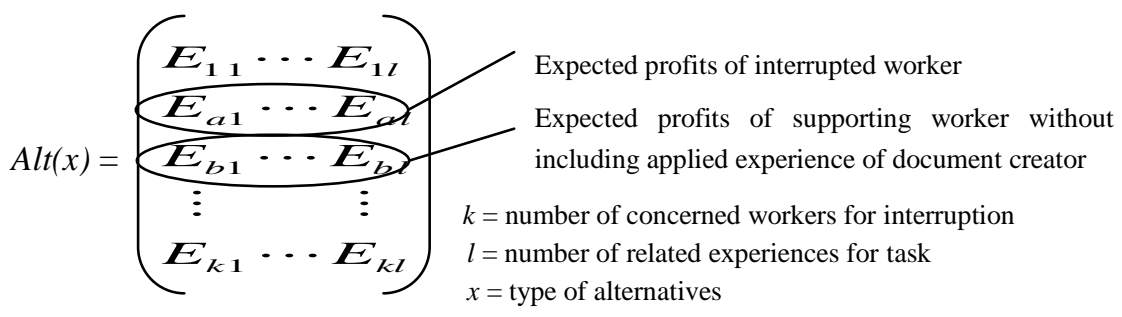

Figure 4

Matrix of alternatives

The formulated alternatives $(\operatorname{Alt}(x))$ are ranked by computing scores $(C)$ of TOPSIS method based on Euclidean distance of the positive ideal solution (TDP) and the negative ideal solution $(T N P)$. Each distance of the interrupted worker $(D P(1))(D N(1))$ and the supporting worker $(D P(2))(D N(2))$ to the positive or the negative ideal solution are computed by computing following expressions.

$$
\begin{array}{ll}
D P(1)=\sqrt{\sum_{i=1}^{l}\left(E_{k l}-E_{a l}\right)^{2}} & D P(2)=\sqrt{\sum_{i=1}^{l}\left(E_{k l}-E_{b l}\right)^{2}} \\
D N(1)=\sqrt{\sum_{i=1}^{l}\left(E_{k l}^{\prime}-E_{a l}\right)^{2}} & D N(2)=\sqrt{\sum_{i=1}^{l}\left(E_{k l}^{\prime}-E_{b l}\right)^{2}} \\
T D P=\sqrt{\sum_{i=1}^{k}(D P(k))^{2}} & T D N=\sqrt{\sum_{i=1}^{k}(D N(k))^{2}} \\
C=\frac{T D N}{T D P+T D N} & l=\text { number of related experiences for task } \\
k & k=\text { number of concerned workers for interruption }
\end{array}
$$




\section{Case Model of Interruption in Office Work}

Interruption of workflow is occurred by overlapping workflows of collaborative work in office work. Concerned workers to the interrupted workflow must improve work efficiency to achieve related tasks. Overmuch improvement must be avoided to keep expected nurture for pre-assigned workers. A system development work is operated with sharing tasks by multiple workers as collaborative work. Workers who have similar role participate to workflow to achieve objective by sharing tasks of the target workflow. The participated workers who have history of developing another system are interrupted by trouble shooting according to user inquiry. The generated activity is operated by interrupted worker till resolving the problem. In order to assist decision making for adaptive task selection in this interruption case, the proposed approach is applied to concerned workers in certain interrupted workflow. Assumed situation model for adaptive task selection in interruption is an actual system development of three workers in a Japanese company. Target three workers who have different working history are developing a sales analysis tool in intra network of the company based on assignment of project leader. The sales analysis tool is developed by using Microsoft Excel with VBA (Visual Basic for Applications). The ranking of adaptive task selection is changed according to the situation of interruption and the subjective attributes of interrupted worker. Firstly, subjective attributes of concerned workers as working experience are computed according to existing working report of two month by using pre-weighted impact of tasks. The focused type of experiences consists of type of computer language, user supporting and specification creation and so on. Table 1 shows sample definitions for weighting impact of experience. The subjective attributes of three target workers are calculated according to working report as Table 2. Each score as the past accumulated experiences (We(i)) of each worker in Table 2 is computed by using expression (2)(3) in this paper.

Table 1

Target type of experiences

\begin{tabular}{|c|c|}
\hline Type of experiences & Keywords \\
\hline$W e(1)$ & Visual Basic \\
\hline$W e(2)$ & JavaScript \\
\hline$W e(3)$ & HTML \\
\hline$W e(4)$ & VBA \\
\hline$W e(5)$ & Database \\
\hline$W e(6)$ & MS Excel \\
\hline$W e(7)$ & User supporting \\
\hline$W e(8)$ & Find and fix of bug \\
\hline$W e(9)$ & Specification consideration \\
\hline$W e(10)$ & System updating \\
\hline$W e(11)$ & Document creation \\
\hline
\end{tabular}


Table 2

The subjective attributes of each worker in two month

\begin{tabular}{|c|c|c|c|c|c|c|c|c|c|c|c|}
\hline & $W e(1)$ & $W e(2)$ & $W e(3)$ & $W e(4)$ & $W e(5)$ & $W e(6)$ & $W e(7)$ & $W e(8)$ & $W e(9)$ & $W e(10)$ & $W e(11)$ \\
\hline Worker 1 & 6.88 & 9.50 & 6.75 & 2.93 & 7.59 & 3.60 & 4.02 & 4.36 & 5.91 & 5.89 & 4.65 \\
\hline Worker 2 & 7.38 & 10.92 & 6.74 & 1.00 & 12.30 & 1.00 & 1.00 & 1.00 & 7.38 & 1.00 & 5.09 \\
\hline Worker 3 & 1.00 & 12.13 & 9.17 & 2.71 & 7.18 & 3.82 & 1.67 & 1.00 & 2.44 & 1.00 & 1.00 \\
\hline
\end{tabular}

Worker 1 who has developed first version of the sales analysis tool has experience of web system development. Although Worker 2 has also the experience of the web system development and database development, he is manager who has small experience for VBA development. Worker 3 has experience of supporting development of Worker 1 and he had been trained to develop web application in first month. The positive ideal solution in assumed interruption situation is computed by using each worker's subjective attributes and pre-assignment of tasks. When the interruption is occurred to Worker 1 by adding the task from another workflow as Task 7 due to trouble for another system, operating time for Task 1 and Task 4 of Worker 1 is consumed by generated Task 7 . In order to computing appropriate alternatives for improving negative effect of the interruption to Worker 1 , the positive ideal solution is computed based on selectable pre-assigned tasks to the concerned workers. Table 3 shows relation of workers and the pre-assigned tasks that have same deadline in assumed interrupted situation. The target workers in this company can work eight hour per day.

Table 3

Relation of pre-assigned tasks

\begin{tabular}{|c|c|c|c|}
\hline & Operating time & Core time & Assigned worker \\
\hline Task 1 & 8.0 & 5.0 & Worker 1 \\
\hline Task 2 & 8.0 & 4.0 & Worker 2 \\
\hline Task 3 & 8.0 & 5.0 & Worker 3 \\
\hline Task 4 & 8.0 & 4.0 & Worker 1 \\
\hline Task 5 & 8.0 & 4.0 & Worker 2 \\
\hline Task 6 & 8.0 & 5.0 & Worker 3 \\
\hline Task 7 & 8.0 & 5.0 & nothing \\
\hline
\end{tabular}

Table 4 shows scores of weighted tasks by experienced worker. The weights are scored by using fuzzy membership function according to decision of the experienced worker to each task. Task 1 is bug fix activity for improving duplication of inserting data. Task 2 is changing activity of target database for filtering selectable organization function. Task 3 is designing activity for using the system by web browser. Task 4 is improvement activity for showing data quickly on viewer of the system. Task 5 is changing activity of target database for adding data function. Task 6 is design activity for adding data on web browser. 
Table 4

Weighted target tasks of same deadline in interruption

\begin{tabular}{|c|c|c|c|c|c|c|c|c|c|c|c|}
\hline & $W e(1)$ & $W e(2)$ & $W e(3)$ & $W e(4)$ & $W e(5)$ & $W e(6)$ & $W e(7)$ & $W e(8)$ & $W e(9)$ & $W e(10)$ & $W e(11)$ \\
\hline Task 1 & 0.1 & 0.0 & 0.0 & 0.0 & 0.2 & 0.0 & 0.2 & 0.4 & 0.0 & 0.1 & 0.0 \\
\hline Task 2 & 0.0 & 0.0 & 0.0 & 0.2 & 0.5 & 0.3 & 0.0 & 0.0 & 0.0 & 0.0 & 0.0 \\
\hline Task 3 & 0.0 & 0.1 & 0.5 & 0.0 & 0.1 & 0.0 & 0.0 & 0.0 & 0.2 & 0.0 & 0.1 \\
\hline Task 4 & 0.0 & 0.0 & 0.0 & 0.5 & 0.0 & 0.3 & 0.0 & 0.2 & 0.0 & 0.0 & 0.0 \\
\hline Task 5 & 0.0 & 0.0 & 0.0 & 0.2 & 0.5 & 0.3 & 0.0 & 0.0 & 0.0 & 0.0 & 0.0 \\
\hline Task 6 & 0.0 & 0.1 & 0.4 & 0.0 & 0.1 & 0.0 & 0.0 & 0.0 & 0.3 & 0.0 & 0.1 \\
\hline Task 7 & $\mathbf{0 . 1}$ & $\mathbf{0 . 1}$ & 0.0 & 0.0 & 0.0 & 0.0 & $\mathbf{0 . 4}$ & $\mathbf{0 . 4}$ & 0.0 & 0.0 & 0.0 \\
\hline
\end{tabular}

The negative effect $(E C t)$ of Task7 to Worker 1 is computed based on the above the subjective attributes of Worker 1 and the objective attributes of Task 7.

$$
\begin{aligned}
& \operatorname{SPf}(1)=\frac{(8.0 * 0.1)}{(8.0 * 0.1)+6.88}=0.104 \\
& \operatorname{SPf}(2)=0.078, \operatorname{SPf}(7)=0.443, \operatorname{SPf}(8)=0.423 \\
& i \operatorname{SPf}=(\operatorname{SPf}(1)+\operatorname{SPf}(2)+\operatorname{SPf}(7)+\operatorname{SPf}(8)) / 4=0.262 \\
& E C t=5.0+(i \operatorname{SPf} *(8.0-5.0))=5.79
\end{aligned}
$$

Table 5 shows the computed subjective completion time for each worker according to the subjective attributes of workers and the objective attributes of pre-assigned tasks.

Table 5

Subjective flexible time of pre-assigned tasks

\begin{tabular}{|c|c|c|c|}
\hline & Completion time & Flexible time & Assigned worker \\
\hline Task 1 & 5.66 & 2.34 & Worker 1 \\
\hline Task 2 & 6.09 & 1.91 & Worker 2 \\
\hline Task 3 & 5.78 & 2.22 & Worker 3 \\
\hline Task 4 & 5.66 & 2.34 & Worker 1 \\
\hline Task 5 & 6.09 & 1.91 & Worker 2 \\
\hline Task 6 & 5.82 & 2.18 & Worker 3 \\
\hline
\end{tabular}

Therefore, the exceeding time of interrupted worker (Worker 1) is 1.11 hours. The alternatives for appropriate task selection must improve this exceeding time by supporting of other worker.

$$
E E C t=5.79+(2.34+2.34)=1.11
$$

Figure 5 shows the computed matrix of positive ideal solution according to the above pre-assignment of tasks and target worker's subjective attributes. 


$$
P S=\left(\begin{array}{l}
0.10,0.00,0.00,0.58,0.17,0.40,0.28,0.67,000,0.12,0.00 \\
0.00,0.00,0.00,1.11,0.49,1.29,0.00,0.00,0.00,0.00,0.00 \\
0.00,0.12,0.56,0.00,0.20,0.00,0.00,0.00,0.85,0.00,0.80
\end{array}\right)
$$

Figure 5

Matrix of positive ideal solution by pre-assignment

In order to avoid overmuch improvement for the computed exceeding time of Worker 1, each score of the negative ideal solution is computed by using task assignment that is shortest completion time. There are 90 combinations in this situation that three worker operates two tasks from six tasks. The negative ideal solution in Figure 6 is computed by using the task assignment of shortest completion time within 90 combinations.

$$
N S=\left(\begin{array}{l}
0.10,0.00,0.00,0.58,0.17,0.40,0.28,0.67,000,0.12,0.00 \\
0.00,0.14,0.68,0.00,0.12,0.00,0.00,0.00,0.42,0.00,0.27 \\
0.00,0.00,0.00,0.71,0.70,0.75,0.00,0.00,0.00,0.00,0.00
\end{array}\right)\left(\begin{array}{l}
\text { W1: Task 1, Task 4) } \\
(\mathrm{W} 2: \text { Task 3, Task 6) } \\
(\mathrm{W} 3: \text { Task 2, Task 5) }
\end{array}\right.
$$

Figure 6

Matrix of negative ideal solution

The selectable alternatives that can improve 1.11 hours of Worker 1 are four types of supporting in Figure 7. Task 1 and Task 4 of Worker 1 are selectable by Worker 2 and Worker 3. The required time for each worker is changed by supported tasks. Since Worker 2 and Worker 3 have two tasks, the number of alternatives is changed to eight by changing applied flexible time of tasks.

$$
\begin{array}{ll}
R t(T 1 W 2)=1.11 * 0.41 / 0.22=2.04 & R t(T 4 W 2)=1.11 * 0.71 / 0.42=1.89 \\
R t(T 1 W 3)=1.11 * 0.46 / 0.22=2.33 & R t(T 4 W 3)=1.11 * 0.53 / 0.42=1.42
\end{array}
$$

\section{Figure 7}

Required time for the supported tasks

Table 6 shows each score of alternatives for improving required time of Worker 1 . These scores are used to compute the distance of the positive ideal solution and negative ideal solution as Table 7 shown. The appropriate alternative in this situation is $\operatorname{Alt}(7)$ that Task 4 of Worker 1 is supported by utilizing flexible time of Task 3 of Worker 3. 
Table 6

Scores of alternatives without similar document

\begin{tabular}{|c|c|c|c|c|c|c|c|c|c|c|c|}
\hline & $W e(1)$ & $W e(2)$ & $W e(3)$ & $W e(4)$ & $W e(5)$ & $W e(6)$ & $W e(7)$ & $W e(8)$ & $W e(9)$ & $W e(10)$ & $W e(11)$ \\
\hline \multirow{3}{*}{$\begin{array}{c}\text { Alt(1) } \\
\text { T1 to T2, } \\
\text { T5 of W2 }\end{array}$} & 0.06 & 0.00 & 0.00 & 0.49 & 0.11 & 0.32 & 0.18 & 0.49 & 0.00 & 0.07 & 0.00 \\
\hline & 0.03 & 0.00 & 0.00 & 1.05 & 0.47 & 1.24 & 0.29 & 0.45 & 0.00 & 0.17 & 0.00 \\
\hline & 0.00 & 0.12 & 0.56 & 0.00 & 0.20 & 0.00 & 0.00 & 0.00 & 0.85 & 0.00 & 0.80 \\
\hline \multirow{3}{*}{$\begin{array}{c}\text { Alt(2) } \\
\text { T1 to T5, } \\
\text { T2 of W2 }\end{array}$} & 0.06 & 0.00 & 0.00 & 0.49 & 0.11 & 0.32 & 0.18 & 0.49 & 0.00 & 0.07 & 0.00 \\
\hline & 0.03 & 0.00 & 0.00 & 1.04 & 0.47 & 1.22 & 0.29 & 0.45 & 0.00 & 0.17 & 0.00 \\
\hline & 0.00 & 0.12 & 0.56 & 0.00 & 0.20 & 0.00 & 0.00 & 0.00 & 0.85 & 0.00 & 0.80 \\
\hline \multirow{3}{*}{$\begin{array}{l}\text { Alt(3) } \\
\text { T1 to T3 of } \\
\text { W3 }\end{array}$} & 0.06 & 0.00 & 0.00 & 0.49 & 0.11 & 0.32 & 0.18 & 0.49 & 0.00 & 0.07 & 0.00 \\
\hline & 0.00 & 0.00 & 0.00 & 1.11 & 0.49 & 1.29 & 0.00 & 0.00 & 0.00 & 0.00 & 0.00 \\
\hline & 0.19 & 0.11 & 0.49 & 0.00 & 0.23 & 0.00 & 0.22 & 0.48 & 0.78 & 0.19 & 0.73 \\
\hline \multirow{3}{*}{$\begin{array}{c}\text { Alt(4) } \\
\text { T1 to T6 of } \\
\text { W3 }\end{array}$} & 0.06 & 0.00 & 0.00 & 0.49 & 0.11 & 0.32 & 0.18 & 0.49 & 0.00 & 0.07 & 0.00 \\
\hline & 0.00 & 0.00 & 0.00 & 1.11 & 0.49 & 1.29 & 0.00 & 0.00 & 0.00 & 0.00 & 0.00 \\
\hline & 0.19 & 0.11 & 0.50 & 0.00 & 0.23 & 0.00 & 0.22 & 0.48 & 0.77 & 0.19 & 0.73 \\
\hline \multirow{3}{*}{$\begin{array}{c}\text { Alt(5) } \\
\text { T4 to T2 of } \\
\text { W2 }\end{array}$} & 0.08 & 0.00 & 0.00 & 0.44 & 0.13 & 0.27 & 0.22 & 0.51 & 0.00 & 0.09 & 0.00 \\
\hline & 0.00 & 0.00 & 0.00 & 1.39 & 0.44 & 1.49 & 0.00 & 0.27 & 0.00 & 0.00 & 0.00 \\
\hline & 0.00 & 0.12 & 0.56 & 0.00 & 0.20 & 0.00 & 0.00 & 0.00 & 0.85 & 0.00 & 0.80 \\
\hline \multirow{3}{*}{$\begin{array}{c}\text { Alt(6) } \\
\text { T4 to T5 of } \\
\text { W2 }\end{array}$} & 0.08 & 0.00 & 0.00 & 0.44 & 0.13 & 0.27 & 0.22 & 0.51 & 0.00 & 0.09 & 0.00 \\
\hline & 0.00 & 0.00 & 0.00 & 1.38 & 0.44 & 1.48 & 0.00 & 0.27 & 0.00 & 0.00 & 0.00 \\
\hline & 0.00 & 0.12 & 0.56 & 0.00 & 0.20 & 0.00 & 0.00 & 0.00 & 0.85 & 0.00 & 0.80 \\
\hline \multirow{3}{*}{$\begin{array}{c}\text { Alt(7) } \\
\text { T4 to T3 of } \\
\text { W3 }\end{array}$} & 0.08 & 0.00 & 0.00 & 0.44 & 0.13 & 0.27 & 0.22 & 0.51 & 0.00 & 0.09 & 0.00 \\
\hline & 0.00 & 0.00 & 0.00 & 1.11 & 0.49 & 1.29 & 0.00 & 0.00 & 0.00 & 0.00 & 0.00 \\
\hline & 0.00 & 0.11 & 0.52 & 0.21 & 0.18 & 0.10 & 0.00 & 0.22 & 0.81 & 0.00 & 0.76 \\
\hline \multirow{3}{*}{$\begin{array}{c}\text { Alt( }(8) \\
\text { T4 to T6 of } \\
\text { W3 }\end{array}$} & 0.08 & 0.00 & 0.00 & 0.44 & 0.13 & 0.27 & 0.22 & 0.51 & 0.00 & 0.09 & 0.00 \\
\hline & 0.00 & 0.00 & 0.00 & 1.11 & 0.49 & 1.29 & 0.00 & 0.00 & 0.00 & 0.00 & 0.00 \\
\hline & 0.00 & 0.11 & 0.52 & 0.21 & 0.18 & 0.10 & 0.00 & 0.22 & 0.81 & 0.00 & 0.76 \\
\hline
\end{tabular}

Table 7

Scores and ranking of alternatives without similar document

\begin{tabular}{|c|c|c|c|c|}
\hline & $T D P$ & $T D N$ & $C$ & Ranking \\
\hline Alt(1) & 0.624 & 2.625 & 0.8080 & No.5 \\
\hline Alt(2) & 0.626 & 2.613 & 0.8067 & No.6 \\
\hline Alt(3) & 0.659 & 2.623 & 0.7993 & No.7 \\
\hline Alt(4) & 0.660 & 2.619 & 0.7987 & No.8 \\
\hline Alt(5) & 0.512 & 2.854 & 0.8479 & No.4 \\
\hline Alt(6) & 0.504 & 2.846 & 0.8495 & No.3 \\
\hline Alt(7) & 0.420 & 2.512 & 0.8572 & No.1 \\
\hline Alt(8) & 0.421 & 2.519 & 0.8569 & No.2 \\
\hline
\end{tabular}

However, the ranking of alternatives are changing by applying similar documentation to the supporting workers. When the documents that are written by target workers can be utilized as Table 8 shown, the similarity for the supported tasks is computed to shorten required time for the supporting workers. 
Table 8

Proportion of term frequency for documents

\begin{tabular}{|c|c|c|c|c|c|c|c|c|c|c|c|}
\hline & $W e(1)$ & $W e(2)$ & $W e(3)$ & $W e(4)$ & $W e(5)$ & $W e(6)$ & $W e(7)$ & $W e(8)$ & $W e(9)$ & $W e(10)$ & $W e(11)$ \\
\hline $\begin{array}{c}\text { D1 } \\
\text { Writen by } \\
\text { Worker 1 }\end{array}$ & 0.0 & 0.0 & 0.0 & 0.0 & 0.5 & 0.0 & 0.1 & 0.3 & 0.0 & 0.1 & 0.0 \\
\hline $\begin{array}{c}\text { D2 } \\
\text { Writen by } \\
\text { Worker 2 }\end{array}$ & 0.4 & 0.0 & 0.2 & 0.0 & 0.2 & 0.2 & 0.0 & 0.0 & 0.0 & 0.0 & 0.0 \\
\hline $\begin{array}{c}\text { D3 } \\
\text { Writen by } \\
\text { Worker 3 }\end{array}$ & 0.0 & 0.5 & 0.2 & 0.0 & 0.3 & 0.0 & 0.0 & 0.0 & 0.0 & 0.0 & 0.0 \\
\hline
\end{tabular}

Table 9

Computed similarity of tasks and documents

\begin{tabular}{|c|c|c|}
\hline & Type of Documents & Smilarity \\
\hline \multirow{3}{*}{ Task 1 } & D1 & $\mathbf{0 . 8 2}$ \\
\cline { 2 - 3 } & D2 & 0.30 \\
\hline \multirow{3}{*}{ Task 2 } & D3 & 0.19 \\
\cline { 2 - 3 } & D1 & $\mathbf{0 . 6 8}$ \\
\cline { 2 - 3 } & D2 & 0.49 \\
\hline \multirow{3}{*}{ Task 3 } & D3 & 0.39 \\
\cline { 2 - 3 } & D1 & 0.15 \\
\hline \multirow{3}{*}{ Task 4 } & D2 & 0.40 \\
\cline { 2 - 3 } & D3 & $\mathbf{0 . 5 2}$ \\
\cline { 2 - 3 } & D1 & 0.16 \\
\hline \multirow{3}{*}{ Task 5 } & D2 & $\mathbf{0 . 1 8}$ \\
\cline { 2 - 3 } & D3 & 0.00 \\
\cline { 2 - 3 } & D1 & $\mathbf{0 . 6 8}$ \\
\hline \multirow{3}{*}{ Task 6 } & D2 & 0.49 \\
\cline { 2 - 3 } & D3 & 0.39 \\
\cline { 2 - 3 } & D1 & 0.16 \\
\hline
\end{tabular}

The required time for supporting workers is changed by applying most similar document. Since the similarity of D1 to Task 1 in Table 9 is high and the experiences of document creator (Worker 1) is high, the required time for Task 1 is reduced longer than Task 4.

$$
\begin{array}{ll}
\operatorname{Rt}(T 1 W 2)=1.11 * 0.19 / 0.22=0.95 & \text { Rt }(T 4 W 2)=1.11 * 0.67 / 0.42=1.80 \\
\operatorname{Rt}(T 1 W 3)=1.11 * 0.20 / 0.22=1.00 & \operatorname{Rt}(T 4 W 3)=1.11 * 0.51 / 0.42=1.36
\end{array}
$$

Figure 8

Required time for the supported tasks with similar document

Table 10 shows changed ranking of alternatives by applying similar document. Despite the score of experience for Worker 3 to Task 1 and Task 4 is higher than Worker 2, the supporting of Worker 2 to Task 1 is best score of alternatives in this situation. 
Table 10

Scores and ranking of alternatives with similar document

\begin{tabular}{|c|c|c|c|c|}
\hline & $T D P$ & $T D N$ & $C$ & Ranking \\
\hline Alt(1) & 0.415 & 2.616 & 0.8632 & No.1 \\
\hline Alt(2) & 0.416 & 2.609 & 0.8626 & No.2 \\
\hline Alt(3) & 0.417 & 2.612 & 0.8624 & No.3 \\
\hline Alt(4) & 0.417 & 2.611 & 0.8622 & No.4 \\
\hline Alt(5) & 0.501 & 2.848 & 0.8504 & No.8 \\
\hline Alt(6) & 0.494 & 2.840 & 0.8519 & No.7 \\
\hline Alt(7) & 0.411 & 2.524 & 0.8599 & No.5 \\
\hline Alt(8) & 0.412 & 2.522 & 0.8596 & No.6 \\
\hline
\end{tabular}

\section{Conclusions}

The scores of positive ideal solution and negative ideal solution of TOPSIS method is adjusted to the interrupted workflow by aggregating subjective attributes of the worker and objective attributes of the task in this paper. In addition, flexibility of score for alternatives is also extended by applying similar documentation. The proposed approach has indicated that experienced worker is not always appropriate supporter in the improvement of certain interruption. However, even if there are alternatives to get higher profit for the organization with improving the negative impact of interruption, the proposed approach recommends alternatives to the interrupted worker based on the assignment of the superior decision maker. Therefore, the alternatives for assisting decision making in certain interruption by the proposed approach cannot optimize the objective of the pre-assignment by the estimation of the superior decision maker according to the objective of organization. Since the time of the mental work for gathering information from experiences of the worker is increased due to a forgetting with time, the subjective attributes of the worker in this study is not adjusted to actual values by computing reduction of memory for worker's experiences. In order to improve accuracy for computing ranking of alternatives, the impact on shortening task operation with documents must be evaluated by comparing the proposed cosine distance with another data searching approaches. Although the risk of interruption is based on the operation time consumed for the workflow in certain time, uncertain context of interruption must be reasoned for more appropriate supporting the decision making of the interrupted worker as future work. To measure the amount of change by interruption, we provide to handle the interruptions on the running workflow, and how such change fits in the subjective and objective situation of the interrupted worker. This can be represented as fitness function which the direction of our future works. In order to reason the uncertain context of interruption, the predicting approaches based on the other worker's subjective attributes than the proposed experience should be discussed in the future work by using fitness function method, safety measure approach and so on. 


\section{References}

[1] Herbert A. Simon, The Science of the Artificial, The MIT Press third edition edition (1996)

[2] L. Corragio, Deleterious Effects of Intermittent Interruptions on the Task Performance of Knowledge Workers, A Laboratory Investigation, Unpublished doctoral dissertation, University of Arizona (1990)

[3] M. Al-Yakoob, H. D. Sherali, Mixed Integer Programming Models for an Employee Scheduling Problem with Multiple Shifts and Work Locations, Annals of Operational Research 155 (2007) pp. 119-142

[4] N. Moradi nasab, R. Shafaei, M. Rabiee, M. Mazinani, Minimization of Maximum Tardiness in a No-Wait TwoSstage Flexible Flow Shop, International Journal of Artificial Intelligence 8 (2012) pp. 166-181

[5] R. J. Wherry, The Curve of Forgetting: Its Statistical Application, Journal of Educational Psychology, 23 (1932) pp. 621-624

[6] Cheri Speier, Joseph S. Valacich, Iris Vessey, The Influence of Task Interruption on Individual Decision Making: An Information Overload Perspective, Decision Sciences 30 (1999)

[7] Amos Tversky, Daniel Kahneman, Judgment under Uncertainty: Heuristics and Biases, Science, New Series 185 (1974) pp. 1124-1131

[8] K. Sugawara, Decision Support System for Handling Interruption in Tasks for Workers, New Trends in Software Methodologies Tools and Techniques Proceedings of the $11^{\text {th }}$ SoMeT_12 (2012) pp. 273-281

[9] T. L. Saaty, The Analytical Hierarchy Process, New York: McGraw-Hill (1980)

[10] G. H. Tzeng, C. H. Chiang, C. W. Li, Evaluating Intertwined Effects in eLearning Programs: A Novel Hybrid MCDM Model Based on Factor Analysis and DEMATEL, Expert Systems with Applications 32 (2007) pp. 1028-1044

[11] S. Greco, B. Matarazzo, R. Slowinski, Rough Sets Methodology for Sorting Problems in Presence of Multiple Attributes and Criteria, European Journal of Operational Research 138 (2002) pp. 247-259

[12] K. Yoon, C. L. Hwang, Multiple Attribute Decision Making Methods and Applications. Springer Verlag, Berlin, Germany (1980)

[13] S. Opricovic, Multicriteria Optimization of Civil Engineering Systems, Faculty of Pennsylvania (1998)

[14] S. J. Chen, C. L. Hwang, Fuzzy Multi Attribute Decision Making, Lecture Notes in Economics and Mathematical system series 375, Springer Verlag, New York (1992) 
[15] Z. Yue, An Extended TOPSIS for Determining Weights of Decision Makers with Interval Numbers, Knowledge-Based Systems 24 (2011) pp. $146-153$

[16] J. Tian, D. Yu, B. Yu, S. Ma, A Fuzzy TOPSIS Model via Chi-Square Test for Information Source Selection, Knowledge-Based Systems 37 (2013) pp. $515-527$

[17] E. Pap, V. Bojanic, M. Georgijevic, G. Bojanic, Application of PseudoAnalysis in the Synchronization of Container Terminal Equipment Operation, Acta Polytechnica Hungarica 8 (2011) pp. 5-21

[18] Albert Corominas, JordiOlivellan, RafaelPastor, A Model for the Assignment of a Set of Tasks When Work Performance Depends, Int. J. Production Economics 126 (2010) pp. 335-340

[19] G. Salton and C. Buckley, Term-Weighting Apploarches in Automatic Text Retrieval, Information Processing \& Management 24 (1988) pp. 513-523 